\title{
MODELACIÓN ESPACIAL MEDIANTE GEOMÁTICA Y EVALUACIÓN MULTICRITERIO PARA LA ORDENACIÓN TERRITORIAL
}

\section{SPATIAL MODELING BY MEANS OF GEOMATIC AND MULTICRITERIA EVALUATION FOR TERRITORIAL ARRANGEMENT}

\author{
Carlos Mena Frau ${ }^{1} \quad$ John Gajardo Valenzuela ${ }^{2} \quad$ Yony Ormazábal Rojas $^{2}$ \\ Recibido el 5 de agosto de 2005, aceptado el 16 de enero de 2006 \\ Received: August 5, 2005 Accepted: January 16, 2006
}

\begin{abstract}
RESUMEN
Los encargados de la planificación territorial se enfrentan al problema de manejar una gran cantidad de información espacial que les permita cumplir su labor en forma oportuna y satisfactoria. La presente investigación plantea una metodología basada en la integración de la Geomática y las Técnicas de Evaluación Multicriterio (EMC) para obtener un modelo de capacidad de acogida que facilite la localización de sitios adecuados para albergar un relleno sanitario. La zona de estudio corresponde a las Comunas de Longaví, Retiro y Parral, ubicadas en la VII Región del Maule-Chile. Se construyó una base de datos digital vectorial que incluyó: red caminera, hidrografía, curvas de nivel, pendientes, orientaciones, clases y permeabilidad de suelos, cubierta vegetacional, asentamientos urbanos, entre otras. De la superficie analizada sólo un 1,86\% incluye a las zonas de "muy alta" y "alta" capacidad, un 2,61\% representa a zonas de "media" capacidad, y un 95,53\% de la superficie modelada abarca las clasificaciones de "baja", "muy baja" y "excluyente", quedando en evidencia el reducido porcentaje de superficie apto para seleccionar sitios idóneos. La metodología desarrollada puede ser utilizada como una herramienta de apoyo a la toma de decisiones en los procesos de planificación territorial, acotando la superficie sobre la cual se deben realizar estudios más intensivos, pudiendo ser replicada en otros escenarios y enriquecida a través de la inclusión de nuevos criterios y variables que se ajusten mejor a la realidad estudiada. El programa contó con financiamiento del Fondo Nacional de Desarrollo Regional (FNDR) VII Región.
\end{abstract}

Palabras clave: Evaluación multicriterio, geomática, planificación territorial, modelo de capacidad de acogida, relleno sanitario.

\section{ABSTRACT}

The territorial planning managers facing the problem of to handle a great amount of space information that it allows them to fulfill his work in opportune and satisfactory form. The present investigation raises a methodology based on the integration of Geomatic and Techniques of Multicriteria Evaluation to obtain a welcome capacity model that facilitates the location of adapted sites to lodge a sanitary filling. The zone of study corresponds to the Communes of Longaví, Retiro and Parral, located in VII Region of Maule - Chile. A vectorial digital data base was constructed, including: road network, hydrography, contours, ground slopes, directions, classes and permeability, vegetation cover, urban establishments, among others. Only $1,86 \%$ of the analyzed surface includes zones of "very high" and "high" capacity, 2,61\% represents zones of "medium" capacity, and 95,53\% of the modeled surface includes classifications of "loss", "very low" and "excluding", being left in evidence the reduced percentage of apt surface to select suitables sites. The developed methodology can be used as a tool of support to taking decision in the processes of territorial planning, limiting the surface on which more intensive studies are due to make, being able to be talked back in other places and to be enriched through the inclusion of new criteria and variables that adjust better to the studied reality. The program counted on financing of the Regional Development National Fund (FNDR) VII Region.

Keywords: Multicriteria evaluation, geomatica, territorial planning, welcome capacity model, sanitary filling.

\footnotetext{
1 Universidad de Talca, Facultad de Ciencias Forestales, Departamento de Gestión Forestal Ambiental. Avenida Lircay s/n. Talca, Chile. e-mail: cmena@utalca.cl.

2 Universidad de Talca, Facultad de Ciencias Forestales, Departamento de Gestión Forestal Ambiental. Avenida Lircay s/n. Talca, Chile. e-mail: semsig@utalca.cl.
} 


\section{INTRODUCCIÓN}

Uno de los problemas más apremiantes que enfrenta hoy el medio ambiente, resultado directo de la propia evolución de la actual sociedad, es el aumento en la producción de residuos. Las actividades que el hombre realiza son de muy diversa índole y naturaleza, y en función de las mismas, generan distintos tipos de residuos de diversa composición, estado o peligrosidad. De acuerdo a estas características, el tratamiento, gestión y almacenamiento de los distintos residuos varían, dando lugar a la necesidad de crear toda una serie infraestructuras y mecanismos de gestión, con el fin de evitar cualquier deterioro ambiental [1].

La localización en el territorio de las instalaciones que realizan estas funciones es un problema de gran importancia y fuerte contenido geográfico. De acuerdo a Maass y Plata [2], desde un punto de vista espacial, el problema se puede esquematizar de la siguiente manera: las instalaciones donde se tratan y depositan los residuos constituyen hechos de tamaño puntual (en términos relativos); los traslados de residuos entre los lugares de producción y tratamiento se realizan generalmente a través de la red vial existente; finalmente, la población, junto con otros elementos sensibles, se sitúa concentrada en muchos puntos del territorio, existiendo, por lo tanto, una distribución espacial irregular.

En este contexto, el problema geográfico se resume en contestar la siguiente pregunta: ¿En qué punto o puntos se deben colocar las instalaciones para que atiendan de modo adecuado a la población y/o a las empresas encargadas de la gestión y recolección de los residuos? [3].

Actualmente se ha optado por la utilización de la Geomática, que básicamente es la integración de múltiples tecnologías, como son los Sistemas de Información Geográfica (SIG), la Teledetección aérea y espacial, y los Sistemas de Posicionamiento Global (GPS), como una herramienta para el manejo y gestión de bases de datos geográficas y territoriales. No obstante, a pesar de las múltiples ventajas de procesamiento, consulta y almacenamiento de información que estos sistemas poseen, no son una novedad las limitaciones que presentan a la hora de realizar labores de análisis y modelación territorial. En este sentido, la respuesta y una posible solución a estas limitaciones pueden darse en la integración de métodos externos al ámbito de la Geomática, entre los que se encuentran las Técnicas de Evaluación Multicriterio (EMC) [4].
En consecuencia, el objetivo del presente estudio fue establecer una metodología para la localización de rellenos sanitarios sobre la base del uso de la Geomática y las Técnicas de Evaluación Multicriterio, apoyado por la generación de un modelo de capacidad de acogida para las comunas de Longaví, Retiro y Parral, pertenecientes a la Provincia de Linares en la VII Región del Maule, Chile (ver figura 1).

\section{CUERPO}

El estudio fue desarrollado empleando una metodología experimental que utiliza múltiples técnicas de la Geomática como una herramienta para la captura, construcción y gestión de una base de datos digital de la zona de estudio. Posteriormente, se integraron con dichos métodos las Técnicas de Evaluación Multicriterio (EMC), dando un carácter cuantitativo a las evaluaciones realizadas. Este aspecto ya había sido comentado anteriormente por Pullar [5], quien indicaba las ventajas de la evaluación sobre datos espacialmente relacionados. En este contexto, el proceso se dividió en tres grandes fases: la primera tiene que ver con la recopilación de los datos cartográficos, estudios anteriores y la construcción de la base de datos digital, incluyendo las transformaciones necesarias para la utilización de los temas geográficos como variables espaciales. La segunda fase incluye las actividades desarrolladas para la evaluación de las variables incluidas y la consulta al grupo de profesionales participantes. Finalmente, en la tercera fase, se obtiene el modelo de capacidad preliminar, para posteriormente, y a través de labores de verificación en terreno, obtener el modelo de capacidad de acogida definitivo.

\section{A) Primera Fase}

- Recopilación de Información Base: Se recopiló información cartográfica, temática y estadística emanada de estudios realizados en el sector comprendido por la investigación, considerando información cartográfica del Instituto Geográfico Militar (IGM), ya sea en formato analógico y digital; coberturas digitales de las comunas involucradas, proporcionadas por la Secretaría de Planificación y Cooperación (Serplac) VII Región del Maule; información digital de suelos CIREN-CORFO; diagnósticos comunales, marco legal y normativa relacionada al tratamiento y disposición de los residuos sólidos domiciliarios. 


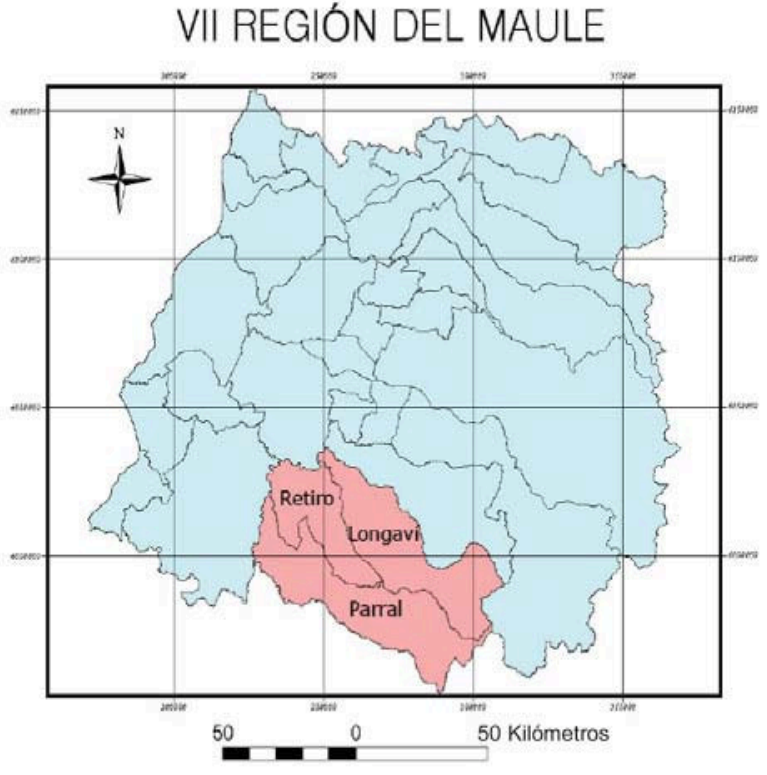

Fig. 1 Zona de Estudio.

- Determinación de variables: Las variables debían cumplir con la condicionante de la representatividad espacial, por lo que se consideraron las siguientes: uso actual y permeabilidad del suelo, distancia a los caminos y vías de acceso (accesibilidad), pendiente del terreno, distancia al centro productor de residuos (transporte), red hidrográfica, vegetación, áreas de conservación y áreas turísticas.

- Definición de las restricciones asociadas: Corresponden a un cierto tipo de criterio que restringe en forma permanente la disponibilidad del sitio para la evaluación de las alternativas [9]. Bajo este prisma, la definición de las restricciones asociadas incluyó: distancia hacia cursos de agua, ocupación de suelos agrícolas productivos, proximidad de los sitios a los centros urbanos y sectores poblados, proximidad a los caminos y superficie del terreno.

- Rasterización de la base de datos digital: El modelo de capacidad de acogida se generó en formato ráster, específicamente en el software IDRISI, por lo cual resultó necesario hacer compatibles las coberturas con este tipo de formato. En este contexto, la rasterización permite transformar archivos vectoriales en archivos ráster de Idrisi. Posteriormente, estos archivos pasaron a formar parte de las coberturas ráster con las cuales se construyó el modelo de capacidad de acogida.

- Definición de las alternativas: Las alternativas están representadas por objetos o unidades espaciales, las cuales representan un sitio individual, constituyendo así el conjunto global de alternativas de selección [9]. A continuación, se exponen las clases temáticas (alternativas de selección) utilizadas en cada criterio de la investigación:

- Permeabilidad de los suelos: Se agruparon los rangos de permeabilidad de los suelos utilizando el método del número de curvas, comúnmente empleado en el cálculo de escorrentías superficiales [10]. Dicho método supone las siguientes clases de suelos: Grupo I (A), Grupo II (B), Grupo III (C), Grupo IV (D).

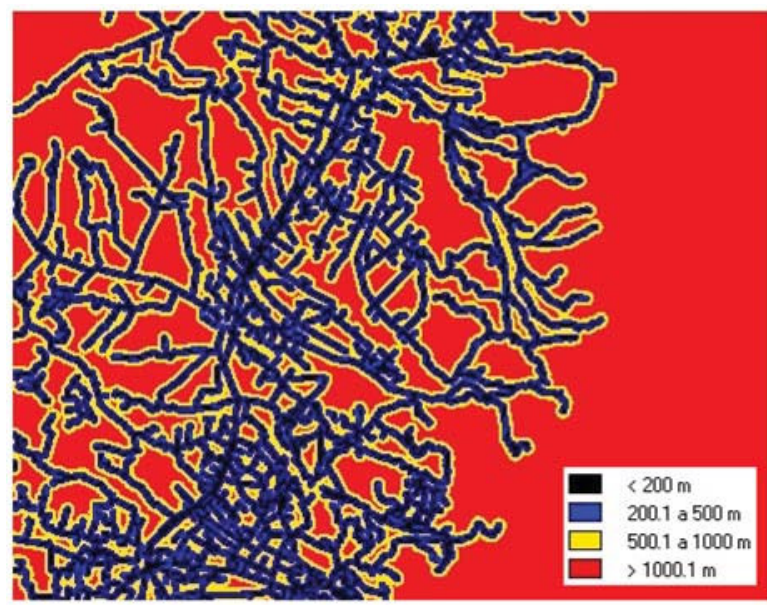

Fig. 2 Distancia a los caminos y vías de acceso.

- Clases de suelos: Se consideró el uso actual del suelo y, más aún, su clase y capacidad para acoger actividades productivas y agroeconómicas. De esta forma, la cobertura estuvo conformada por las siguientes clases temáticas: Clase I, Clase II, Clase III, Clase IV, Clase V, Clase VI, Clase VII, Clase VIII.

- Distancia al centro productor de residuos (Distancia de transporte): Se utilizó una cobertura que representaba las distancias hacia los centros urbanos y hacia las agrupaciones pobladas. Posteriormente, se reagrupó la cobertura resultando las clases que indican los siguientes rangos de distancia: 0,6 a $15 \mathrm{~km} ; 15$ a 30 km; 30 a 45 km; 45 km y más.

- Distancia a los caminos y vías de acceso (Accesibilidad): Se construyó una cobertura de distancias, tomando como referencia las principales vías y caminos de la zona de estudio. Agrupando las distancias, resultaron los siguientes rangos: 200 a 500 metros; 500 a 1.000 metros; 1.000 metros y más (ver figura 2). 
- Pendientes y exposiciones: La utilización de las pendientes y exposiciones del terreno involucraron la creación de un Modelo Digital del Elevaciones (MDE) (ver figura 3). No obstante, toda utilización de un MDE implica una validación que debe realizarse en terreno. Con este objeto, se recolectaron puntos en terreno con equipos GPS geodésicos, para luego realizar una comparación de éstos con sus homólogos en el MDE. De acuerdo con el Federal Geographic Data Committee (FGDC), el parámetro más apropiado para describir la precisión de este tipo de modelos es el error Cuadrático Medio o Root Mean Square (RMS), el cual describe la variación global entre una serie de puntos medidos en el "mundo real" y sus correspondientes en el MDE [11]. Su expresión analítica es:

$$
R M S=\sqrt{\frac{\sum\left(Z T_{i}-M D E_{i}\right)^{2}}{n}}
$$

donde:

$$
\begin{aligned}
& \mathbf{Z T}_{\mathbf{i}} \quad \text { : altitud medida sobre el terreno con } \\
& \text { MDE }_{\mathbf{i}} \quad \text { : altitud medida sobre el MDE en el }
\end{aligned}
$$

Así, para la escala 1:50.000 sobre la cual está basada la confección del MDE, se puede establecer un RMS máximo aceptable de $14 \mathrm{~m}$, considerando los RMS establecidos por el FGDC y el US Geological Survey (USGS) para las escalas citadas [12]. En la recolección de puntos, se utilizó un receptor GPS Geodésico de alta precisión. A partir de estos puntos se llevó a cabo el cálculo del RMS, obteniendo un valor de $12,78 \mathrm{~m}$, con un error máximo de $29 \mathrm{~m}$. Considerando que el error RMS del MDE final se encontraba por debajo del valor medio y debido a los aspectos antes comentados, se pudo establecer que la fiabilidad del MDE era aceptable, según los parámetros máximos de RMS indicados por el FGDC y el USGS.

En la confección de la cobertura de pendientes, se utilizó una función de superficie que permitió crear una cobertura de pendientes a partir del MDE ya generado, resultando las siguientes clases temáticas: 0 a $15 \%$ de inclinación; 15 a $30 \%$ de inclinación; 30 a $45 \%$ de inclinación;
$45 \%$ de inclinación y más. La siguiente capa derivada fue la de orientaciones, la cual debió ser "filtrada" para corregir ciertas angulosidades e imperfecciones [13], obteniendo finalmente las siguientes alternativas de selección: norte, este, sur, oeste y llano o sin exposición.

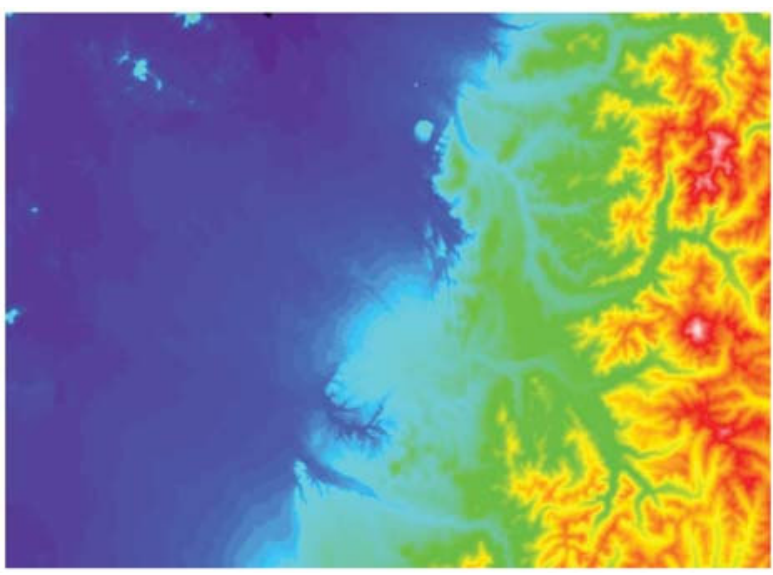

Fig. 3 Modelo digital del terreno para las comunas de Longaví, Retiro y Parral.

- Inclusión de las restricciones: Las restricciones representan las alternativas que contienen sólo dos valores numéricos: el código 1 , que indica aquel lugar candidato para cierta actividad, y el valor 0 , que indica la anulación total de dicho sector para la actividad evaluada. A continuación, se mencionan las restricciones aplicadas y los sectores que fueron considerados como potenciales o restrictivos:

- Proximidad hacia cursos de agua superficiales: Los sectores con una distancia superior a 1.000 metros respecto a los cursos de agua, se codificaron con el valor 1 . Por otro lado, sectores cuya distancia hacia los cursos de agua fue inferior a 1.000 metros, se codificaron con el valor 0 (ver figura 4).

- Clase de los suelos: Los polígonos pertenecientes a las clases I, II, y III, se codificaron con el valor 0 . El resto de los polígonos identificados con las clases IV, V, VI, VII y VIII, se codificaron con el valor 1, indicando que esas zonas eran candidatas a ser evaluadas.

- Proximidad hacia los centros urbanos y poblados: Las distancias inferiores a 600 metros con respecto a los polígonos que conforman los centros urbanos se codificaron con el valor 0 ; se asignó valor 1, a aquellas distancias 
superiores a 600 metros de los polígonos anteriormente mencionados.

- Vegetación, especies protegidas y espacios sensibles: Se designaron los espacios que presentaban especies contenidas en el Libro Rojo de la Flora Chilena. También se incluyeron zonas legalmente excluidas, tales como: bosques nativos de protección, matorrales y renovales de protección.

- Proximidad hacia los caminos: La clase excluyente se fijó para distancias inferiores o iguales a 200 metros con respecto a los caminos, adoptando sus pixeles la codificación 0 . La siguiente categoría codificada con el valor 1 indicó distancias superiores a 200 metros con respecto a los caminos, determinándose como posibles zonas para ser incluidas en la evaluación.

- Superficie del terreno: Se excluyeron aquellos polígonos que no cumplían con la mínima superficie requerida. De este forma se discriminaron todos aquellos polígonos cuya área no cumpliera con un mínimo de aproximadamente 12 hectáreas.

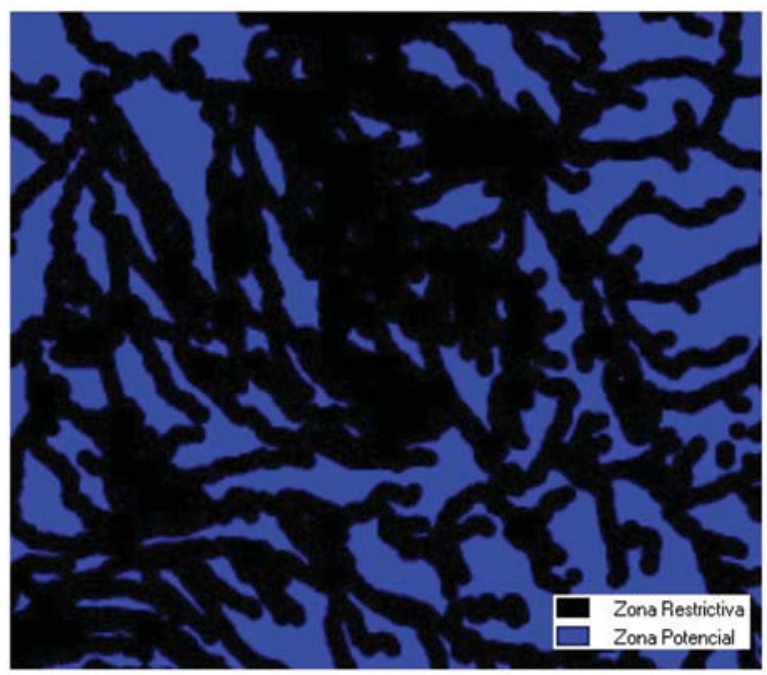

Fig. 4 Restricción de proximidad a cursos de agua.

\section{B) Segunda Fase}

- Técnicas de Evaluación Multicriterio: La aplicación de estas técnicas en el ámbito de la Geomática requiere que cada variable o factor temático deben ser estructurados como una matriz, en la cual los criterios ocupan las filas de la matriz y las alternativas propias de cada criterio ocuparán las columnas [6]. En el presente estudio, se utilizó el Método de las Jerarquías Analíticas (MAJ) propuesto por Saaty [14]. La finalidad fue comparar la importancia de uno de los factores sobre cada uno de los demás $\left(\mathrm{a}_{\mathrm{ij}}\right)$, para posteriormente determinar el eigenvector principal, el cual establece los pesos $\left(\mathrm{w}_{\mathrm{j}}\right)$; y el eigenvalor, que proporciona una medida cuantitativa de la consistencia de los juicios de valor entre pares de factores [7]. La asignación de los juicios de valor fue realizada apoyándose en la opinión y entrevistas con expertos en el tema del estudio. El universo de profesionales consultados agrupó a los siguientes organismos: Universidad de Talca, Gobierno Regional del Maule, Municipalidades de Retiro, Longaví y Parral, Empresas de Servicios RSU, CONAMA, Servicio de Salud del Maule.

- La Sumatoria Lineal Ponderada: Se optó por la utilización del método de la Sumatoria Lineal Ponderada, aplicado frecuentemente en modelos desarrollados en Sistemas de Información Geográfica que trabajan con datos ráster [8]. La ecuación que describe el método de la Sumatoria Lineal Ponderada es la siguiente:

$$
r_{i}=\sum_{i=1}^{n}\left(w_{j} * e_{i j}\right)
$$

donde:

$\mathbf{r}_{\mathbf{i}}$ : capacidad del terreno para la ubicación de rellenos sanitarios.

$\mathbf{w}_{\mathbf{j}}$ : peso del criterio $\mathbf{j}$.

$\mathbf{e}_{\mathrm{ij}}$ : valor normalizado de la alternativa $\mathrm{i}$ en el criterio j.

n : número de criterios involucrados en la investigación.

Los valores $\left(r_{i}\right)$ más altos indican sectores potencialmente adecuados para la ubicación del relleno sanitario. La aplicación de las restricciones (distancia a cursos de agua, distancia a caminos, distancia a núcleos poblados, clases de suelos, vegetación de interés, superficie mínima) se realizó a través de un proceso de álgebra de mapas, en donde cada restricción o capa ( $1 / 0)$ es multiplicada con la cobertura que contiene los valores de capacidad de acogida $\left(r_{i}\right)$, extrayendo de esta forma las zonas restrictivas. Una vez realizada las operaciones de álgebra de mapas, se obtuvo el modelo de capacidad de acogida preliminar. 


\section{C) Tercera Fase}

- Verificación del modelo de capacidad de acogida: Fue necesario realizar la validación del modelo de capacidad en terreno. Dicha validación se llevó a cabo con la ayuda de un receptor GPS geodésico, el cual permitió llegar en forma precisa a sectores preestablecidos. Una vez allí, se realizó una inspección visual, identificando posibles elementos y situaciones que presentaran problemas al seleccionar un sitio como posible candidato. De esta forma, si a través de las observaciones se concluía que un sector presentaba serios inconvenientes para albergar un relleno sanitario, se codificaba apropiadamente el polígono que lo representaba y posteriormente, se eliminaba del modelo. Asimismo, si se concluía que el sector no presentaba aparentes problemas, se codificaba dicho polígono como un sitio potencialmente apto para albergar un relleno sanitario. De este proceso, se obtuvo una cobertura validada en terreno y clasificada en seis categorías de capacidad de acogida (Muy alta, Alta, Media, Baja, Muy Baja y Excluyente).

\section{D) Resultados y Discusión}

Una vista general del modelo de capacidad de acogida obtenido se puede apreciar en la figura 5. La figura es resultado del desarrollo de una metodología que incluye la integración de la Geomática y las Técnicas de Evaluación Multicriterio, constituyendo una primera aproximación para los posibles estudios de prefactibilidad que se desarrollen sobre los sitios identificados. Las categorías de capacidad de acogida obtenidas en el modelo pueden ser visualizadas en la tabla 1. En dicho cuadro se aprecia claramente la gran extensión del terreno que se descarta del análisis (350.543,9 ha), debido a la inclusión de múltiples restricciones en la modelación, y particularmente aquellas relacionadas con la restricción de clase de suelos. En este contexto, es factible pensar que mientras más restricciones espaciales sean incluidas en la modelación, la región factible y las superficies candidatas a ser soluciones, tienden a disminuir.

Con respecto a las categorías del modelo que alcanzaron la puntuación de Muy alta y Alta capacidad, la tabla 1 muestra su reducida superficie $(1,86 \%)$ en comparación con el total de la zona de estudio. Esto se debe a que sólo una pequeña porción del terreno acaparó las puntuaciones más altas en cada alternativa de selección, efecto que resulta de la evaluación realizada por los expertos participantes y se ve reflejado en el modelo, ya que la Sumatoria Lineal Ponderada puede ser considerada un método EMC de carácter compensatorio.
Tabla 1 Categorías, puntaje y superficie para las zonas del modelo de capacidad de acogida.

\begin{tabular}{|l|c|r|c|}
\hline Categoría & Puntuación & $\begin{array}{c}\text { Superficie } \\
\text { (ha) }\end{array}$ & $\begin{array}{c}\text { Porcentaje } \\
\text { del total }\end{array}$ \\
\hline Muy Alta & $80-98$ & $3.854,2$ & 0,97 \\
\hline Alta & $60-80$ & $3.542,8$ & 0,89 \\
\hline Media & $40-60$ & $10.331,7$ & 2,61 \\
\hline Baja & $20-40$ & $1.935,8$ & 0,49 \\
\hline Muy Baja & $0-20$ & $26.011,9$ & 6,57 \\
\hline Excluyente & - & $350.543,9$ & 88,47 \\
\hline
\end{tabular}

Los sectores obtenidos en el modelo se presentan por comunas, con una descripción generalizada de los aspectos naturales y del entorno, haciendo hincapié en los aspectos positivos o negativos que presentan. En la tabla 2, se aprecian los sitios obtenidos para la comuna de Longaví. En su mayoría se trata de terrenos agrícolas, relativamente planos, utilizados generalmente para el cultivo de arroz y otros cereales. No obstante, existen ciertos sectores en los cuales los terrenos se encuentran poblados por matorrales esclerófilos y son usados para el pastoreo y talaje de animales. En general, los accesos a los sectores se encuentran disponibles durante todo el año; sin embargo, para acceder en su totalidad a los sitios se debe considerar el mejoramiento de algunos tramos que no se encuentran en buen estado.

En la tabla 3, se aprecian los sitios obtenidos para la comuna de Retiro; en su mayoría se trata de terrenos agrícolas, y dependiendo del cultivo éstos presentan en algunas ocasiones numerosos cursos de agua superficiales, generalmente canales.

Los accesos a los sectores se encuentran en buen estado, en algunas ocasiones las vías se encuentran asfaltadas. No obstante, como inconveniente destacan las distancias que los camiones tendrían que recorrer para transportar los residuos.

En la tabla 4, es posible apreciar los sectores resultantes del modelo para la comuna de Parral. En su mayoría, y al igual que los casos anteriores, corresponden a sitios agrícolas, de topografía plana, utilizados para el cultivo preferencial de arroz y trigo.

Por tratarse de terrenos agrícolas usados para cultivos anuales, generalmente, cuentan con vías de acceso en buen estado, cuyas carpetas se encuentran estabilizadas. Sin embargo, presentan algunos inconvenientes como la gran cantidad de canales de regadíos que en algunas ocasiones se presentan en los sitios o en sus inmediaciones, esto debido a que la mayoría de los cultivos que sustentan requieren de elevados aportes del recurso hídrico. 
Tabla 2 Sectores y descripción de lugares candidatos en la Comuna de Longaví.

\begin{tabular}{|l|l|}
\hline Sector & Descripción \\
\hline Cerro & $\begin{array}{l}\text { Distante a } 18 \text { km de Longaví, ubicado } \\
\text { en la falda sur del cerro Mesamávida, } \\
\text { presenta matorrales y especies } \\
\text { esclerófilas nativas (espinos, litres, } \\
\text { boldos, entre otros). El sitio presenta } \\
\text { un gran número de cárcavas, no se } \\
\text { aprecian cursos de agua superficiales } \\
\text { cercanos y el camino de acceso se } \\
\text { encuentra en buen estado. }\end{array}$ \\
\hline $\begin{array}{l}\text { Bellavista } \\
\text { (ver figura }\end{array}$ & $\begin{array}{l}\text { Distante 15 km de Longaví. Terreno } \\
\text { agrícola usado para el cultivo de arroz } \\
\text { y cereales. El suelo muestra una } \\
\text { textura arcillosa y su topografía es } \\
\text { plana, no presenta cursos de agua } \\
\text { superficiales y el camino de acceso L- } \\
\text { 480 se encuentra ripiado y en buen } \\
\text { estado. }\end{array}$ \\
\hline Los Cristales & $\begin{array}{l}\text { Distante 15 km de Longaví, es un } \\
\text { sector con terrenos de uso } \\
\text { preferentemente agrícola, se aprecia } \\
\text { trigo y en algunas extensiones maíz y } \\
\text { huertos de consumo. Los terrenos no } \\
\text { utilizados en cultivo se emplean como } \\
\text { praderas y pastoreo de animales. En } \\
\text { las cercanías del sector se aprecia la } \\
\text { presencia del Estero Tengue. }\end{array}$ \\
\hline
\end{tabular}

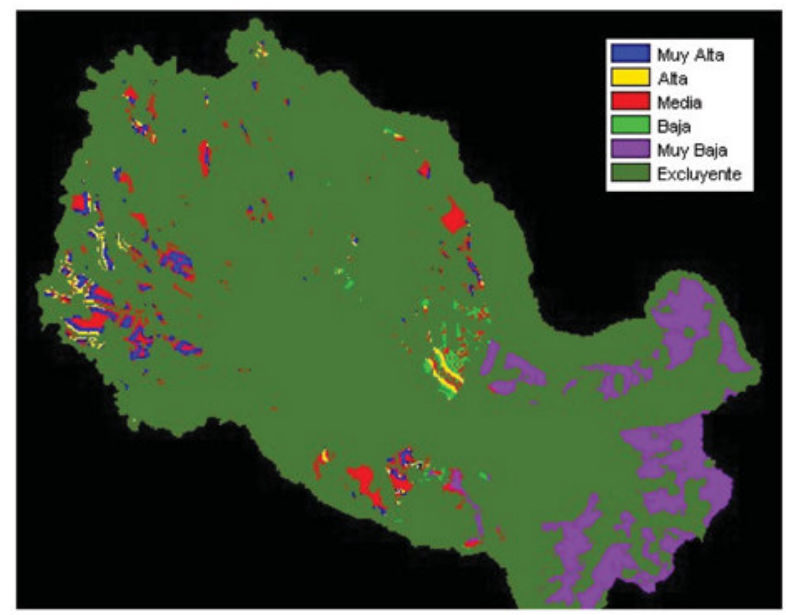

Fig. 5 Modelo de capacidad de acogida para las comunas de Longaví, Retiro y Parral.
Tabla 3 Sectores y descripción de lugares candidatos en la Comuna de Retiro.

\begin{tabular}{|l|l|}
\hline Sector & Descripción \\
\hline La Granja & $\begin{array}{l}\text { Distante aproximadamente 8 km de } \\
\text { Retiro. Terreno relativamente plano, } \\
\text { usado en el cultivo de cereales, se } \\
\text { observan ciertas áreas empleadas } \\
\text { como praderas. El camino de acceso } \\
\text { al sector se encuentra recientemente } \\
\text { estabilizado no obstante, en las } \\
\text { cercanías del sector se aprecia una } \\
\text { pequeña cantidad de viviendas. }\end{array}$ \\
\hline $\begin{array}{l}\text { Las Rosas } \\
\text { (ver figura }\end{array}$ & $\begin{array}{l}\text { Distante aproximadamente 8 km de } \\
\text { Retiro, es un terreno relativamente } \\
\text { plano, usado en el cultivo de cereales } \\
\text { y arroz. El sitio se encuentra poblado } \\
\text { de matorrales esclerófilos, entre los } \\
\text { que destaca la Acacia caven. Los } \\
\text { accesos al sector se encuentran en } \\
\text { óptimo estado y no presenta cursos de } \\
\text { agua cercanos. }\end{array}$ \\
\hline La Florida & $\begin{array}{l}\text { Sector distante 36 km de la ciudad de } \\
\text { Retiro. Es un terreno } \\
\text { topográficamente plano, empleado } \\
\text { para el cultivo de especies agrícolas, } \\
\text { esencialmente arroz. El sector } \\
\text { presenta accesos en buen estado, no } \\
\text { obstante en las cercanías presenta } \\
\text { numerosos canales de regadíos y un } \\
\text { curso de agua importante, el estero } \\
\text { Cabo Verde. }\end{array}$ \\
\hline
\end{tabular}

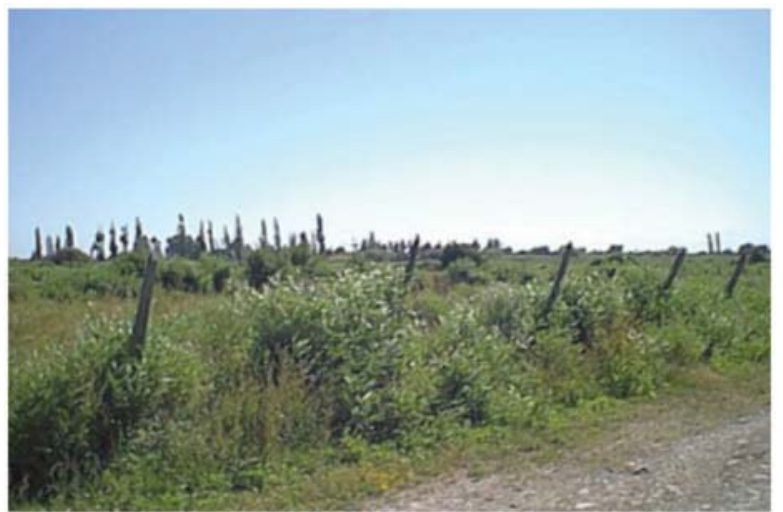

Fig. 6 Sector Bellavista. 


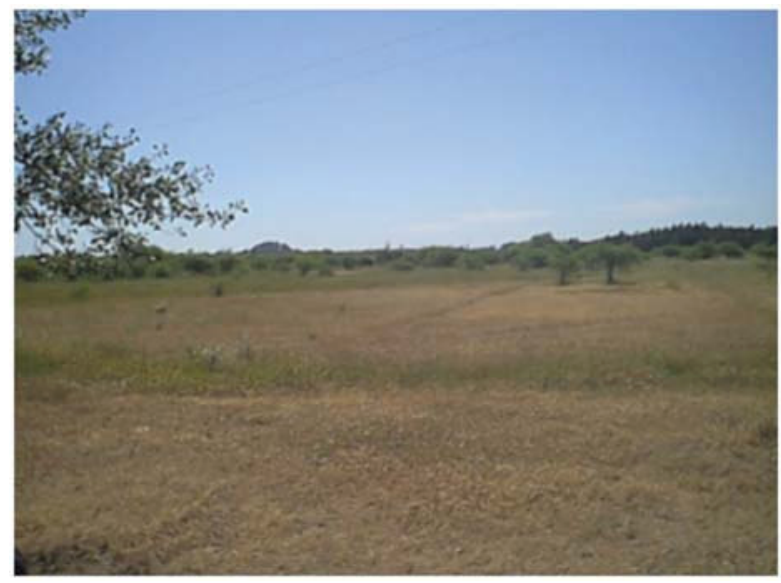

Fig. 7 Sector Las Rosas.

Tabla 4 Sectores y descripción de lugares candidatos en la Comuna de Parral.

\begin{tabular}{|l|l|}
\hline Sector & Descripción \\
\hline $\begin{array}{l}\text { Cuchumillaco figura } \\
\left.\mathrm{N}^{\circ} 8\right)\end{array}$ & $\begin{array}{l}\text { Distante } 26 \mathrm{~km} \text { de Parral, sector } \\
\text { relativamente plano, presenta } \\
\text { matorrales esclerófilos y actualmente } \\
\text { utilizado para pastoreo. Las vías de } \\
\text { acceso se encuentran en buen estado, } \\
\text { no se observan cursos de agua } \\
\text { superficiales en las cercanías. }\end{array}$ \\
\hline Toltón & $\begin{array}{l}\text { Distante 25 km de Parral, los terrenos } \\
\text { del sector son empleados para el } \\
\text { cultivo de arroz, dejando el predio } \\
\text { para el pastoreo una vez que se ha } \\
\text { cosechado la producción. Los } \\
\text { caminos presentan carpeta de ripio y } \\
\text { proveen acceso durante todo el año. } \\
\text { No presenta cursos de agua } \\
\text { superficiales cercanos. Los más } \\
\text { próximos son el río Perquilauqén y el } \\
\text { estero Cuyulemu. }\end{array}$ \\
\hline Titinvilo & $\begin{array}{l}\text { Distante 13 km de Parral, terreno } \\
\text { agrícola empleado actualmente para } \\
\text { el cultivo de trigos, de topografía } \\
\text { plana, presenta accesos ripiados en } \\
\text { buen estado y en sus cercanías existen } \\
\text { algunos canales de regadío que } \\
\text { sustentan los cultivos. }\end{array}$ \\
\hline Lucumilla & $\begin{array}{l}\text { Distante 14 km de Parral, terreno } \\
\text { plano, actualmente se ha dejado en } \\
\text { barbecho y se aprecia algunos } \\
\text { animales. Los accesos son ripiados y } \\
\text { se encuentran en buen estado, no se } \\
\text { aprecian cursos de agua en las } \\
\text { cercanías del sector. }\end{array}$ \\
\hline
\end{tabular}

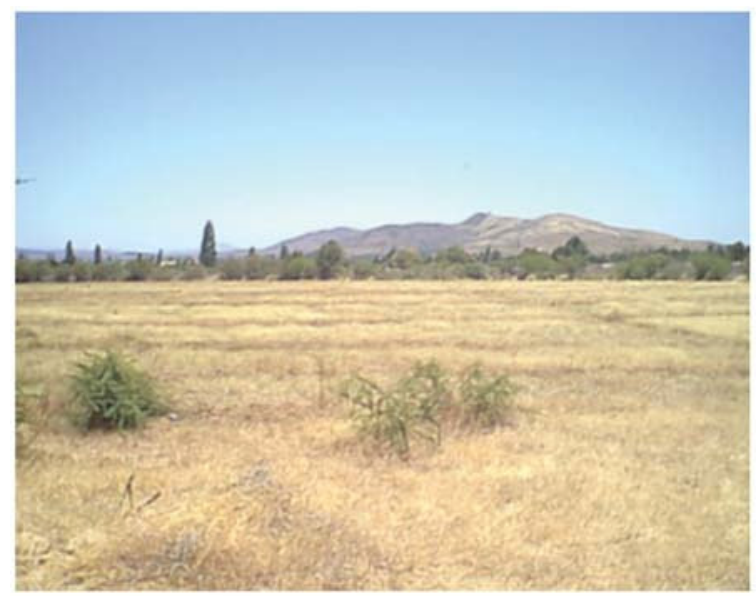

Fig. 8 Sector Cumillaco.

\section{CONCLUSIONES}

- La Geomática, integrada con las técnicas EMC, adquiere gran importancia en estudios de análisis territorial. De hecho, aplicar la sumatoria lineal ponderada en problemas de objetivo simple y múltiples criterios, como ocurre en el presente caso, genera valiosa información que ayuda a la toma de decisiones, particularmente en problemas de asignación de actividades y gestión territorial.

- Los sectores señalados como candidatos, aunque son el resultado de la múltiple evaluación de los expertos consultados y cumplen con la integración de las variables incluidas en el estudio, responden principalmente a dos criterios incluidos en la evaluación: accesibilidad de los sitios y distancia de transporte de los residuos.

- La mayoría de los sitios candidatos corresponden a terrenos agrícolas, cuya topografía indica terrenos relativamente planos, utilizados actualmente para cultivos agrícolas, generalmente de arroz y trigo. Los sitios poseen accesos en buen estado, correspondiendo generalmente a caminos ripiados, que permiten un acceso expedito durante todo el año. Sin embargo, es importante considerar que, para acceder de manera completa a estos sitios, en algunas ocasiones, se deben recorrer huellas que en la mayoría de las veces no se encuentran habilitadas para el tránsito de vehículos pesados durante todo el año. Un punto relevante es que la totalidad de los sectores seleccionados son propiedad de particulares, por lo que la utilización de estos sitios para las actividades de implantación de un relleno sanitario 
implicaría realizar gestiones de adquisición de los terrenos por parte de las municipalidades involucradas.

- Finalmente, es importante destacar que la metodología planteada permite ser extrapolada a otros municipios y escenarios, pudiendo involucrar nuevas variables que permitirán enriquecer cada vez más los resultados obtenidos. Sin embargo, resulta vital que quienes están encargados de tomar decisiones territoriales conozcan y tomen conciencia de la Geomática y las Técnicas de Evaluación Multicriterio como una base técnica de gran valor para estudios de planificación y gestión territorial.

\section{REFERENCIAS}

[1] J. Bosque, M. Gómez, V. Rodríguez, M. Díaz, A. Rodríguez y A. Vela. "Localización de centros de tratamiento de residuos: una propuesta metodológica basada en un SIG". Anales de Geografía de la Universidad Complutense. España. Vol. 19, pp. 295-323. 1999.

[2] S. Maass, R. Plata. "Aplicación de los SIG para la localización asignación de instalaciones no deseables". IX Reunión Nacional SELPER. Zacatecas, México. 1998.

[3] J. Bosque, S. Maass. "Modelos de localizaciónasignación y evaluación multicriterio para la localización de instalaciones no deseables". Serie Geográfica $\mathrm{N}^{\circ}$ 5. Universidad de Alcalá de Henares. Madrid, España, pp. 97-112. 1995.

[4] R. Tkach, S. Simonovic. "A new approach to multi-criteria decision making in water resources". Journal of Geographic Information and Decision Analysis. Canada. Vol. 1. $\mathrm{N}^{\circ}$ 1, pp. 25-44. 1997.

[5] D. Pullar. "Using an allocation model in Multiple criteria evaluation". Journal of Geographic Information and Decision Analysis. Canada. Vol. 3. $\mathrm{N}^{\circ}$ 2, pp. 9-177. 1999
[6] J. Barredo. "Los SIG en la Ordenación del Territorio: Posibilidades y desarrollo utilizando evaluación Multicriterio". En Sistemas de Información Geográfica y Teledetección Espacial Aplicadas a la Ordenación del Territorio y el Medio Ambiente. Universidad de Talca. Talca, Chile, pp. 105-115. 1999.

[7] P. Jankowski. "Integrating geographical information systems and multiple criteria decision-making methods". International Journey Geographical Information Systems. London, United Kingdom. Vol. 9. № 3, pp. 251-273. 1995.

[8] J. Barredo, J. Bosque J. "Multicriteria evaluation methods for ordinal data in a GIS environment". Geographical Systems. USA. Vol. 5, pp. 313-327. 1998.

[9] J. Barredo. "Sistemas de Información Geográfica y Evaluación Multicriterio en la Ordenación del Territorio”. Madrid: Ra-Ma. España, pp. 263. 1996.

[10] V. Chow, D. Maidment, L. Mays. "Hidrología aplicada”. McGraw-Hill. Santafé de Bogotá, Colombia, pp. 584. 1994.

[11] Federal Geographic Data Committe. "Geospatial Positioning Accuracy Standards. Part 3: Nacional Standard for Spatial Data Accuracy". FGDCSTD-007.1-1998. Washington D.C. USA, pp. 28. 1998.

[12] C. Mena. "Análisis del Proceso de Forestación Artificial en la VII Región del Maule: Un Ejemplo Modélico en la Comuna de Empedrado". Tesis para optar al grado de doctor. Alcalá de Henares: Universidad de Alcalá de Henares. España, pp. 358. 1996.

[13] J. Bosque. "Sistemas de Información Geográfica”. Madrid: Rialph S.A. España, pp. 419. 1992.

[14] T. Saaty. "The Analytical Hierarchy Process". New York: Mc Graw Hill. USA, pp. 281. 1980. 